\title{
VALOR NUTRITIVO DE LA PULPA DE CAFÉ SOMETIDA A FERMENTACIÓN SÓLIDA USANDO Aspergillus niger EN POLLOS Y CERDOS1
}

\author{
Mario Molina ${ }^{2}$, Otto Raúl Lechuga y Ricardo Bressani
}

\section{COMPENDIO}

Pulpa de café fue sometida a fermentación sólida usando Aspergillus niger, humedad inicial $80 \%, \mathrm{pH} 3-5,35^{\circ} \mathrm{C}$ y adicionando $2-5 \%$ de urea comercial y $2.0 \%$ de fosfato dicálcico comercial, por $48 \mathrm{~h}$. El material fermentado, secado al sol, probó tener un contenido de polifenoles, cafeína y fibra significativamente menor $(\mathrm{P}<0.05)$ que la pulpa original secada al sol. Además, la proteína verdadera de la pulpa fermentada $(18 \%)$ probó ser significativamente $(\mathrm{P}<0.01)$ mayor que la del material original (5\%). Al Incorporar la pulpa en niveles de 5, 10 y $15 \%$ en raciones para pollos de engorde en crecimiento se encontró que después de 6 semanas la ración conteniendo $15 \%$ de pulpa fermentada presentó una ganancia de peso $(1.43 \mathrm{~kg})$ y una eficiencia alimentarla (120) significativamente igual a la ración control sin pulpa y mejor $(\mathrm{P}<0,05)$ que la ración conteniendo la pulpa sin fermentar a igual nivel de $15 \%$ ( $1.19 \mathrm{~kg}$ y 2.55 , respectivamente). En el caso de cerdos en crecimiento alimentados por 8 semanas con raciones conteniendo el $20 \%$ de ambas pulpas se encontraron similares resultados. Se concluye que la fermentación sólida representa una alternativa tecnológica viable para mejorar el valor nutricional de la pulpa de café. nutrición.

Palabras clave adicionales: Alimento para pollos y cerdos,

\section{INTRODUCCIÓN}

La posible utilización de la pulpa del café en alimentación animal ha sido investigada desde ya hace años. A través de tales investigaciones se ha podido establecer que la pulpa decafé posee factores anti-nutricionales (o tóxicos) que limitan su uso en la alimentación animal, particularmente de animales monogástricos. Se ha podido establecer que dentro de tales factores los más nocivos son cafeína, polifenoles y el alto contenido de fibra de la pulpa (Bressani, 1979). Consecuentemente, se han investigado alternativas para detoxificar este material que representa el subproducto, más abundante del beneficiado del café (Braham, 1979; Molina el al, 1974).

Una alternativa plausible ha comprobado ser el proceso de fermentación sólida usando Aspergillus niger. A través de este proceso se ha podido reducir significativamente $(\mathrm{P}<0.01)$ los niveles de cafeína, polifenoles y fibra de la pulpa, a la vez que su contenido de proteína verdadera (representada por aminoácidos totales) se aumenta de un 5\% a un $15 \%$ (Peñaloza et al, 1985). Por lo mismo, pareció de interés determinar el

\begin{abstract}
Coffee pulp was subjected to a solid-state fermentation process using Aspergillus niger, an Initial moisture content of $80 \%, \mathrm{pH}$ $3.5,35^{\circ} \mathrm{C}$ and adding $2.5 \%$ commercial urea and $2.0 \%$ commercial dicalcium phosphate, for a total of $48 \mathrm{~h}$. The sundried fermented material proved to have a significantly $(\mathrm{P}<0.05)$ lower polyphenolic, caffeine and fiber content than the original sun-dried coffee pulp. Further, the true protein content of the fermented material (18\%) was significantly $(\mathrm{P}<0.01)$ higher than that of the original material $(5 \%)$. When the pulp was included at a 5, 10 and $15 \%$ levels In growing chicken rations It was found that after six weeks the ration containing $15 \%$ of the fermented pulp presented a weight gain $(1.43 \mathrm{~kg})$ and a feed efficiency (2.20) significantly equal to the control ration (without pulp) and better $(\mathrm{P}<0.05)$ than the ration containing the sundried, unfermented pulp at $15 \%$ (1.19 $\mathrm{kg}$ and 2.55 , respectively). In the case of growing swine fed with rations containing either fermented or unfermented pulp at $20 \%$ level for 8 weeks the results were similar to those obtained In the chicken experiment. It is concluded that solid-state fermentation represents a viable technological alternative to improve the nutritional value of coffee pulp.
\end{abstract}

valor nutritivo del material cuando forma parte de raciones para pollos y cerdos en crecimiento.

\section{MATERIALES Y MÉTODOS}

La pulpa de café usada en el presente estudio fue obtenida en un beneficio de Amatitlán (Guatemala). La misma fue recolectada el mismo día de su procesamiento y secada al sol en las facilidades del INCAP. Una vez seca la pulpa fue separada en dos lotes. Un lote fue sometido a fermentación sólida, mientras que el otro fue almacenado para usarse como pulpa sin fermentar. Ambos lotes fueron molidos a 60 mallas. La fermentación sólida se efectuó colocando $68.4 \mathrm{~kg}$ de pulpa ajustada al $80 \%$ de humedad y agregada con urea $(2.5 \%)$ y fósfato dicálcico $(2.0 \%)$, ambos de calidad comercial, en bandejas de $1.0 \times 1.2 \times 0.1 \mathrm{~m}$ con fondo de malla plástica de 60 mallas. Esta pulpa fue inoculada con 2 x 107 esporas viables de A niger y el material fue incubado en

${ }^{1}$ Presentado en la XXXV Reunión Anual del PCCMCA, San Pedro Sula, Honduras, 1989.

Este estudio fue financiado con fondos provistos por la AID de los Estados Unidos (Proyecto INCAP 550). Pubhcación INCAP E- 1288.

${ }^{2}$ Cientifico, División Ciencias Agrícolas y de Alimentos, INCAP, Apartado Postal 1188, Guatemala City, Guatemala, C.A.

Publicado en Agronomia Mesoamericana Vol. 1 (1990). 
un cuarto a $35^{\circ} \mathrm{C}$ y $80 \%$ de humedad relativa, por un total de $48 \mathrm{~h}$. Al final de este tiempo, el material fue secado al sol y molido a 60 mallas en un molino de martillos (Rivera, 1988).

Tanto la pulpa fermentada como la no fermentada fueron sometidas a análisis proximal, evaluaciones de aminoácidos totales, cafeína, polifenoles y fraccionamiento de paredes celulares (AOAC, 1975; Gómez-Brenes y Bressani, 1973; Ishler y Cal., 1948; Van Soest, 1963; Van Soest y Wine, 1967,1968).

Con ambos materiales (pulpa fermentada y sin fermentar), se prepararon dietas para pollos y cerdos (Cuadros 1 y 2, respectivamente). Para el experimento en pollos se usaron 30 pollos por dieta, divididos en tres grupos de diez pollos cada uno. Los pollos se alimentaron con las dietas evaluadas a partir del tercer día de edad hasta la octava semana. La ingestión de alimento y el peso se controló semanalmente. Por separado, se pusieron tres grupos con la dieta control adicionada con la cafeína, la ceniza y una mezcla de estos dos más el ácido tánico, contenidos en la dicta con $15 \%$ de pulpa no fermentada (Cuadro 1). Las condiciones experimentales fueron en general similares a las usadas en estudios previos (Bressani y González, 1978).

El experimento en cerdos se efectuó usando un total de 6 animales (de 6 a 7 semanas de edad), por dieta. Los cerdos fueron alimentados individualmente, controlándose la ingesta y ganancia de peso semanalmente, por un período total de 8 semanas. Las condiciones experimentales fueron similares a las usadas por Cuevas (1976).

\section{RESULTADOS Y DISCUSION}

La composición de la pulpa fermentada y de la pulpa sin fermentar se presenta en el Cuadro 3. Como puede apreciarse, el proceso de fermentación sólida fue capaz de disminuir los niveles de cafeína, polifenoles y

Cuadro 1. Formulación porcentual de dietas experimentales a utilizarse en el estudio de alimentación de pollos de engorde (Calculadas para $21 \%$ de proteína cruda).

\begin{tabular}{lcccccccccc}
\hline & & \multicolumn{1}{c}{ Dietas No. } \\
Ingredientes & $\mathbf{1}$ & $\mathbf{2}$ & $\mathbf{3}$ & $\mathbf{4}$ & $\mathbf{5}$ & $\mathbf{6}$ & $\mathbf{7}$ & $\mathbf{8}$ & $\mathbf{9}$ & $\mathbf{1 0}$ \\
\hline Harina de soya & 35.00 & 35.00 & 35.00 & 35.00 & 35.00 & 35.00 & 35.00 & 35.00 & 35.00 & 35.00 \\
Fosfato dicálcico & 2.10 & 2.10 & 2.10 & 2.10 & 2.10 & 2.10 & 2.10 & 2.10 & 2.10 & 2.10 \\
Carbonato de calcio & 1.50 & 1.50 & 1.50 & 1.50 & 1.50 & 1.50 & 1.50 & 1.50 & 1.50 & 1.50 \\
Sal yodada & 0.45 & 0.45 & 0.45 & 0.45 & 0.45 & 0.45 & 0.45 & 0.45 & 0.45 & 0.45 \\
Premix-100 Pfizer & 0.55 & 0.55 & 0.55 & 0.55 & 0.55 & r 0.55 & 0.55 & 0.55 & 0.55 & 0.55 \\
DL-Metionina & 0.10 & 0.10 & 0.10 & 0.10 & 0.10 & 0.10 & 0.10 & 0.10 & 0.10 & 0.10 \\
Aceite de algodón & 5.00 & 5.00 & 5.00 & 5.00 & 5.00 & 5.00 & 5.00 & 5.00 & 5.00 & 5.00 \\
Maíz amarillo & 55.30 & 50.30 & 45.30 & 40.30 & 50.30 & 45.30 & 40.30 & 53.20 & 55.16 & 52.27 \\
Pulpa de café: & & & & & & & & & & \\
a. Sin fermentar & - & 5.00 & 10.00 & 15.00 & - & - & - & - & - & - \\
b. Fermentada & - & - & - & - & 5.00 & 10.00 & 15.00 & - & - & - \\
Cenizas de 15 lb de pulpa & - & - & - & - & - & - & - & 2.10 & - & 2.10 \\
Calcina & - & - & - & - & - & - & - & - & 0.14 & 0.14 \\
Acido tánico & - & - & - & - & - & - & - & - & - & 0.79 \\
TOTAL & 100.00 & 100.00 & 100.00 & 100.00 & 100.00 & 100.00 & 100.00 & 100.00 & 100.00 & 100.00 \\
\hline
\end{tabular}

Cuadro 2. Formulación de dietas experimentales a usarse en estudio de engorde de cerdos (expresadas porcentualmente, calculadas para $21 \%$ de proteína cruda).

\begin{tabular}{lccc}
\hline & \multicolumn{3}{c}{ Dieta No. } \\
\cline { 2 - 4 } Ingredientes & $\mathbf{1}$ & $\mathbf{2}$ & $\mathbf{3}$ \\
\hline Harina de soya & 35.00 & 35.00 & 35.00 \\
Fosfato dicálcico & 2.10 & 2.10 & 2.10 \\
Carbonato de calcio & 1.50 & 1.50 & 1.50 \\
Sal yodada & 0.45 & 0.45 & 0.45 \\
Premix-100 Pfizer & 0.83 & 0.83 & 0.83 \\
DL-Metionina & 0.10 & 0.10 & 0.10 \\
Aceite de algodón & 5.00 & 5.00 & 5.00 \\
Maíz amarillo & 55.02 & 35.02 & 35.02 \\
Pulpa de café: & & & \\
a. Sin fermentar & - & 20.00 & - \\
b. Fermentada & - & - & 20.00 \\
TOTAL & 100.00 & 100.00 & 100,00 \\
\hline
\end{tabular}

Cuadro 3. Composición porcentual de la pulpa de café sin fermentar y sujeta a fermentación sólida, usando Aspergillus niger y la tecnología de fermentación por bandeja*

\begin{tabular}{lcc}
\hline \multicolumn{1}{c}{ Componente } & \multicolumn{2}{c}{ Concentración (\%) } \\
& Pulpa sin fermentar & Producto fermentado \\
\hline Extracto etéreo & 2.47 & 1.91 \\
Nitrógeno total & 1.83 & 18.27 \\
Aminoácidos totales & 5.37 & 18.02 \\
Ceniza & 5.97 & 12.84 \\
Potasio & 1.48 & 1.12 \\
Fibra cruda & 23.79 & 16.27 \\
Fibra neutro detergente** & 45.48 & 38.52 \\
Fibra ácido detergente*** & 44.24 & 37.00 \\
Celulosa** & 25.31 & 18.83 \\
Hemicelulosa** & 3.23 & 1.69 \\
Lignina** & 21.54 & 20.80 \\
Cafeína & 0.98 & 0.72 \\
Polifenoles & 5.34 & 1.54 \\
\hline
\end{tabular}

Valores promedio de 10 muestras, aproximadamente Componentes de las paredes celulares 
fibra, componentes que se han citado como los factores anti-nutricionales más importantes de la pulpa de café, cuando ésta se considera para la formulación de raciones para animales monogástricos. Las disminuciones citadas probaron ser significativas $(\mathrm{P}<0.05)$. La disminución en fibra puede explicarse por la caída en celulosa y hemicelulosa, principalmente observada al efectuar el fraccionamiento de las paredes celulares. La proteína verdadera puede observarse que aumenta de un 5 a un $18 \%$.

La ganancia de peso, consumo de alimento y eficiencia alimenticia, obtenidos en el experimento con pollos puede observarse en el Cuadro 4. Como puede notarse, las diferencias entre los grupos alimentados con las raciones con pulpa fermentada y aquellos con las que contenían pulpa sin fermentar, son más evidentes entre los grupos recibiendo la ración mayor concentración de pulpa (15\%). En este caso se evidencia ya, que mientras el grupo recibiendo la ración con $15 \%$ de pulpa fermentada presenta una ganancia de peso y eficiencia alimenticia estadísticamente similar al grupo control, el grupo recibiendo la ración con $15 \%$ de pulpa sin fermentar presenta un comportamiento significativamente $(\mathrm{P}<0.05)$ inferior en ambos parámetros. Estos datos se- ñalan ya un efecto benéfico significativo de la fermentación sólida sobre el valor nutritivo de la pulpa de café.

Al comparar estos datos con los encontrados con, la ración control adicionada con cafeína, o cenizas de pulpa, o una combinación de estos dos componentes más ácido tánico (todos a la concentración encontrada en la ración con pulpa sin fermentar al $15 \%$ ), podemos observar (Cuadro 5), que la dieta de menor calidad resultó ser la dieta adicionada con $0.14 \%$ de cafeína únicamente, indicando que éste es quizás el factor anti-nutricional más importante en la pulpa de café. La adición del ácido tánico (al 0.79\%) y las cenizas de la pulpa (al $2.10 \%$ ), conjuntamente con la cafeína, parecen tener una acción protectora sobre los efectos de esta última, ya que el comportamiento de este grupo fue significativamente mejor $(\mathrm{P}<0.05)$ que el alimentado con la ración conteniendo cafeína (al $0.14 \%$ ) únicamente.

En el caso del experimento en cerdos (Cuadro 6), puede observarse claramente también el efecto benéfico de la fermentación sólida sobre la calidad nutricional de la pulpa del café. En todos los casos es evidente el mayor consumo obtenido en la dieta conteniendo la pulpa fermentada.

Cuadro 4. Ganancia de peso, consumo de alimento y eficiencia alimentarla de pollos de engorde alimentados con raciones conteniendo pulpa de café fermentada y sin fermentar*.

\begin{tabular}{|c|c|c|c|c|c|c|c|}
\hline \multirow{3}{*}{ Parámetro } & \multirow{3}{*}{$\begin{array}{l}\text { Ración } \\
\text { Control }\end{array}$} & \multicolumn{6}{|c|}{ Raciones con pulpa de café } \\
\hline & & \multicolumn{3}{|c|}{ Fermentada } & \multicolumn{3}{|c|}{ Sin Fermentar } \\
\hline & & $5 \%$ & $10 \%$ & $15 \%$ & $5 \%$ & $10 \%$ & $15 \%$ \\
\hline $\begin{array}{l}\text { Consumo de alimento } \\
\mathrm{Ng} / 6 \text { sem.) } \\
\text { Ganancia de peso }\end{array}$ & $2.93 \pm 0.12 * *$ & $3.12 \pm 0.04$ & $3.08 \pm 0.05$ & $3.14 \pm 0.10$ & $3.25 \pm 0.09$ & $3.16 \pm 0.12$ & $3.03 \pm 0.17$ \\
\hline $\begin{array}{l}\mathrm{Ng} / 6 \text { sem. } 1 \\
\text { Eficiencia }\end{array}$ & $1.44 \pm 0.05 \mathrm{ab}$ & $1.50 \pm 0.05 \mathrm{a}$ & $1.40 \pm 0.06 \mathrm{ab}$ & $1.43 \pm 0.09 \mathrm{ab}$ & $1.52 \pm 0.08 \mathrm{a}$ & $1.38 \pm 0.04 \mathrm{ab}$ & $1.19 \pm 0.07 \mathrm{~b}$ \\
\hline alimenticia & $2.03 \pm 0.09 \mathrm{a}$ & $2.08 \pm 0.05 \mathrm{lb}$ & $2.21 \pm 0.09 \mathrm{ab}$ & $2.20 \pm 0.09 \mathrm{ab}$ & $2.14 \pm 0.08 \mathrm{ab}$ & $2.29 \pm 0.03 \mathrm{ab}$ & $2.55 \pm 0.09 \mathrm{c}$ \\
\hline
\end{tabular}

* Evaluado en 30 pollos de engorde por dicta, subdivididos en 3 grupos. Peso inicial promedio $80.9 \mathrm{~g}$.

** Desviación estándar del promedio. Letras diferentes indican diferencias estadísticamente significativas $(\mathrm{P}<0.05)$.

Cuadro 5. Ganancia de peso, consumo de alimento y eficiencia alimenticia de pollos alimentados con la dieta control sola y añadida con cafeína, cenizas de pulpa y ácido tánico*

\begin{tabular}{lcccc}
\hline \multirow{1}{*}{$\begin{array}{c}\text { Parámetro } \\
\text { Estimado }\end{array}$} & $\begin{array}{c}\text { Sin } \\
\text { Ningún } \\
\text { Aditivo }\end{array}$ & $\begin{array}{c}\text { Con 0. 14\% } \\
\text { de } \\
\text { Cafeína }\end{array}$ & $\begin{array}{c}\text { Con 2.10\% } \\
\text { de } \\
\text { Cenizas }\end{array}$ & $\begin{array}{c}\text { Con 0. 14\% Cafeína } \\
\mathbf{2 . 1 0 \%} \text { Cenizas y } \\
\mathbf{0 . 7 9} \text { Acido Tánico }\end{array}$ \\
\cline { 2 - 5 } & $2.93 \pm 0.12 \mathrm{ab}^{* *}$ & $1.82 \pm 0.18 \mathrm{e}$ & $2.91 \pm 0.18 \mathrm{ab}$ & $2.76 \pm 0.08 \mathrm{~b}$ \\
$\begin{array}{l}\text { Consumo de Alimento } \\
\mathrm{Ng} / 6 \mathrm{sem} \text {.) }\end{array}$ & & & & \\
$\mathrm{Ganancia}$ de Peso & $1.44 \pm 0.05 \mathrm{ab}$ & $0.80 \pm 0.12 \mathrm{c}$ & $1.41 \pm 0.18 \mathrm{ab}$ & $1.28 \pm 0.09 \mathrm{ab}$ \\
$\mathrm{Ng} / 6$ sem.) & $2.03 \pm 0.09 \mathrm{a}$ & $2.30 \pm 0.13 \mathrm{bc}$ & $2.07 \pm 0.12 \mathrm{ab}$ & $2.17 \pm 0.10 \mathrm{ab}$ \\
Eficiencia Alimenticia & &
\end{tabular}

Evaluados en 30 pollos por dicta, divididos en 3 grupos. Peso inicial promedio $80.9 \mathrm{~g}$. La cantidad de cafeína, ácido tánico y cenizas de pulpa agregada fue la encontrada en la ración $\mathrm{cm} 15 \%$ de pulpa sin fermentar. Desviación estándar del promedio. Letras diferentes indican diferencias estadísticamente significativas $(\mathrm{P}<0.05)$. 
Cuadro 6. Ganancia de peso, consumo de alimento y eficiencia alimentaria de cerdos alimentados con raciones conteniendo pulpa de café fermentada y sin fermentar

\begin{tabular}{lccc}
\hline & Ración & \multicolumn{2}{c}{ Raciones cm Pulpa (20\%) } \\
\cline { 3 - 4 } Parámetro & Control & Fermentada & Sin Fermentar \\
\hline $\begin{array}{l}\text { Consumo de } \\
\text { alimento } \\
(\mathrm{kg} / 8 \text { sem. })\end{array}$ & $78.56 \pm 9.20 * *$ & $86.77 \pm 8.60$ & $\mathrm{X} 36 \pm 7.50$ \\
$\begin{array}{l}\text { Ganancia de } \\
\text { peso } \\
(\mathrm{kg} / 8 \text { sem. }\end{array}$ & $37.33 \pm 3.37 \mathrm{a}$ & $32-67 \pm 1.89 \mathrm{a}$ & $26.92 \pm 4.48 \mathrm{~b}$ \\
$\begin{array}{l}\text { Eficiencia } \\
\text { alimenticia }\end{array}$ & $2.11 \pm 0.26 \mathrm{a}$ & $02-66 \pm 0.26 \mathrm{~b}$ & $2.88 \pm 0.35 \mathrm{~b}$ \\
\hline
\end{tabular}

*Evaluado en 6 cerdos Landras, peso inicial promedio $17.5 \mathrm{~kg}$. Consumo y ganancia de peso fueron estimados semana $\mathrm{e}$ individualmente. ** Desviación estándar del promedio. Letras diferentes indican diferencia estadísticamente significativa $(\mathrm{P}<0.05)$.

Debido a estos datos, ahora se efectúa un estudio de costos para determinar la viabilidad económica del proceso de fermentación sólida, a fin de proponerlo como una alternativa que permita usar un subproducto agrícola de la región en alimentación animal, sustituyendo un grano como el maíz que puede emplearse para consumo humano.

\section{BIBLIOGRAFIA}

ASSOCIATION OF OFFICIAL ANALYTICAL CHENUSTS (AOAC). 1975. "Official methods of analysis of the AOAC", 12 ed. Association of Official Analytical Chemists, Washington, D.C., U.S.A. 1094 p.

BRAHAM, J.E. 1979. Coffee pulp in other species. In "Coffee pulp: Composition, technology and utilization". J.E. Braham y R. Bressani (Eds.) Publicación IDRC No. 108e, International Development Research Centre, Ottawa, Canadá. p. 51-54

BRESSANL R. 1979. Antiphysiological factors in coffee pulp. In "Coffee pulp: Composition, technology, and utilization". J.E. Braham y R. Bressani (Eds.) Publicación IDRC No.
108 e., International Development Research Centre, Ottawa, Canadá. pp 83-88.

; GONZALEZ, J.M. 1978. Evaluación de la pulpa de café como posible sustituto del maíz en raciones para pollos de carne. Arch. Lationoamer. Nutr. (GUA), 29:208-221.

CUEVAS. R. 1976. Optimización y factibilidad económica del proceso de descafeinización de la pulpa de café. Tesis Mag. Sc. CESNA, GUA., Programa Universidad de San Carlos de Guatemala/1NCAP. 120 p. más anexos.

GOMEZ-BRENES, R.; BRESSANI, R. 1973. Método para la determinación de aminoácidos, aplicable a problemas de suplantación, fitomejoramiento y bioquímica nutricional. Arch. Latioanoamer. Nutr. (GUA), 23:443-464.

ISHLER, N.H.; FINUCANE, T.P.; BORKER, E. 1948. Rapid spectrophotometric determination of caffeine. Anal. Chem. (U.SA.) 20:1162-1166

MOL1NA, M.R.; DE IA FUENTE, G.; BATTEN, M.A.; BRESSANI, R. 1974. Decaffeination: A process to detoxify coffee pulp. I Agric, Food Chem. (U.S.A.), 22:1055-1059.

PEÑALOZA, W.; MOLINA. M.R.; GOMEZ-BRENES. R.; BRESSANI, R. 1985. Solid-state fermentation: An alternative to improve the nutritive value of coffee pulp. Appl. Environ. Microbiol. (U.S.A.), 49:388-393.

RIVERA, J.L 1988. Diseño y evaluación del proceso de fermentación sólida de la pulpa de café. Tesis Ing. Quím., Facultad de Ingeniería, Universidad de San Carlos de Guatemala. 51 p. más anexos.

VAN SOEST, PJ. 1963. Use of detergents in the analysis of fibrous feeds. II. A rapid method for the determination of fiber and lignin. J. Assoc. Off. Anal. Chem. (U.S.A.). 46:829-835

; WINE, R.H. 1967. Use of detergents in the analysis of fibrous feeds, IV. The determination of plant cell-wall constituents. J Assoc. Off. Anal. Chem. (U.S.A.). 50:50-55.

; 1968. Determination of lignin and cellulose in acid detergent fiber with permanganate. J. Assoc. Off. Anal. Chem. (U.S.A.), 51:780-785. 